Trauma Berufskrankh 2016 · [Suppl 3]: 18:S231-S240

DOI 10.1007/s10039-015-0091-8

Online publiziert: 14. Oktober 2015

๑) Springer-Verlag Berlin Heidelberg 2015

CrossMark
Kathleen Hartwich · Stefan Rammelt

UniversitätsCentrum für Orthopädie und Unfallchirurgie,

Universitätsklinikum Carl Gustav Carus der TU Dresden, Dresden, Deutschland

\title{
Talus- und Kalkaneusfrakturen
}

\section{Was gibt es Neues?}

Talus und Kalkaneus sind die bedeutenden knöchernen Bausteine des Rückfußes, deren Achsfehlstellung oder Gelenkinkongruenz erhebliche Folgen für die Biomechanik und Funktion des gesamten Fußes haben. Talus- und Kalkaneusfrakturen gehen nicht selten mit einer posttraumatischen Funktionseinschränkung und sekundären Arthrose einher.

Daher ist ein strukturiertes Behandlungskonzept mit Analyse der Frakturmorphologie und exakter Wiederherstellung der Anatomie unabdingbar. Die Behandlung dislozierter Frakturen von Talus und Kalkaneus gehört zu den größten Herausforderungen in der Unfallchirurgie, und gerade für die Kalkaneusfrakturen hat es wiederholte Paradigmenwechsel gegeben, wobei die Diskussion über die optimale Behandlungsstrategie noch lange nicht abgeschlossen ist [32]. Nach langer Zeit der konservativen und semioperativen Behandlung von Kalkaneusfrakturen folgte eine Reihe von operativen Therapiekonzepten [61]. Neben der seit Längerem als Therapiestandard geltenden übungsstabilen, nicht gelenkübergreifenden lateralen Plattenosteosynthese werden zunehmend perkutane und minimalinvasive Verfahren praktiziert [36, 40]. Die Prinzipien der Behandlung der Talusfrakturen sind weniger kontrovers, jedoch haben auch für die Versorgung dieser Verletzungen neue Implantate die operative Palette bereichert.

\section{Talusfrakturen}

\section{Vorbemerkungen}

Der Talus ist durch seine Beteiligung an 3 essenziellen Gelenken des Fußes, dem oberen, dem vorderen unteren (talonavi- kular) und hinten unterem Sprunggelenk (subtalar), zu über $60 \%$ mit Knorpel überzogen und nimmt eine Schlüsselstelle in der Verbindung zwischen Unterschenkel und Fuß ein. Seine Gefäßversorgung wird hauptsächlich durch ein Rete periostale aus den Anastomosen der A. tibialis posterior, A. tibialis anterior und A. fibularis generiert. Durch das Fehlen von Muskelund Sehneninsertionen erfolgt die Blutversorgung des Taluskörpers hauptsächlich über die Gefäße des Sinus und Canalis tarsi am Talushals sowie über Äste der A. tibialis posterior am Deltaband. Talusfrakturen gehören mit ihrer relativ geringen Prävalenz von $0,3-1,7 \%$ zu den seltenen Verletzungen und gehen zudem häufig mit Begleitverletzungen oder gar Polytraumata einher, da eine erhebliche Energie zur Erzeugung von Talusfrakturen erforderlich ist.

Talusfrakturen können in zentrale und periphere Frakturen unterteilt werden [35]. Während zentrale Talusfrakturen, Frakturen des Taluskörpers und Talushalses, meist Ausdruck hoher Gewalteinwirkung mit einer überproportional häufigen Inzidenz von schweren Begleitverletzungen [42] sind, werden periphere Talusfrakturen (Proc. lateralis/fibularis, Proc. posterior und osteochondrale Frakturen), die eher im Rahmen eines Distorsionstraumas mit einem (Sub-)Luxationsmechanismus im Subtalargelenk entstehen, aufgrund der diskreten radiologischen Zeichen oft übersehen und können zu persistierenden Beschwerden mit rascher Arthroseentwicklung führen. Taluskopffrakturen werden ebenfalls zu den peripheren Frakturen gerechnet und sind Ausdruck einer Gewalteinwirkung auf das Chopart-Gelenk [23, 35].
Insbesondere Frakturen des Proc. lateralis (fibularis) tali, die als sog. „Snowboarder's fracture“ bezeichnet werden, und Frakturen des Proc. posterior tali - aber auch Frakturen des Sustentaculum tali des Kalkaneus - sind meist Ausdruck einer subtalaren Luxation, weswegen nach erfolgreicher geschlossener Reposition subtalarer Luxationen eine Computertomographie (CT) gefordert wird [5, 33]. Eine einfach zu handhabende Klassifikation für alle zentralen und peripheren Talusfrakturen wurde von Marti [23] eingeführt und orientiert sich am prognostisch relevanten Dislokationsgrad.

Die häufigen Talushalsfrakturen werden ebenfalls nach dem Dislokationsgrad nach Hawkins [18], der ursprünglich 3 Typen unterschied und die später von Canale und Kelly [10] um einen Typ IV ergänzt wurden, eingeteilt.

Eine genaue Unterscheidung von Taluskörper- und -halsfrakturen ist nur mittels CT-Diagnostik möglich. Die CT ist bei zentralen und peripheren Talusfrakturen obligat und dient nicht nur zur Verifizierung der Frakturart und Planung der definitiven Versorgung, sondern auch zur Vermeidung von folgenschweren übersehenen peripheren Talusfrakturen [41].

\section{Versorgungsstrategie und neue Entwicklungen}

Ziele der operativen Versorgung von dislozierten Frakturen des Talushalses (Typ Hawkins II-IV) und Taluskörpers (Typ Marti III-IV) sind eine exakte anatomische Wiederherstellung der Gelenkkongruenz und Achsenverhältnisse zur Minimierung des Arthroserisikos und zur Vermeidung posttraumatischer Fehlstellungen sowie die Behandlung von beglei- 

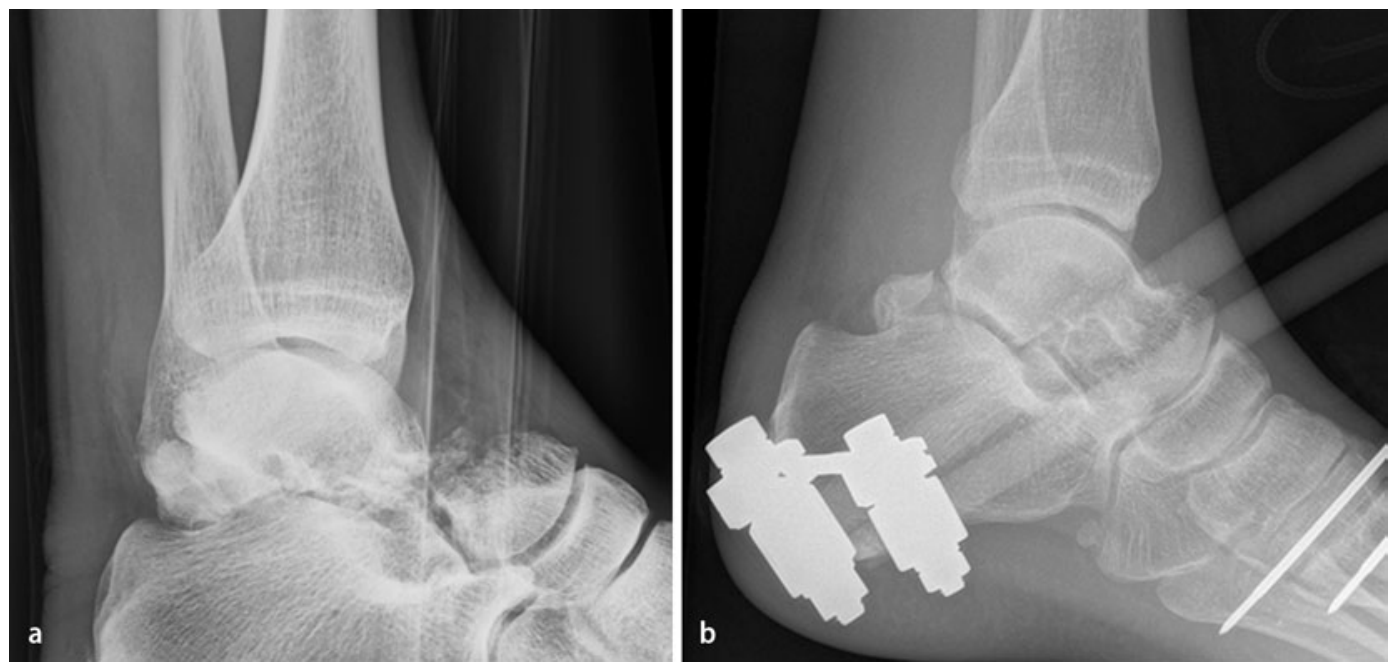

Abb. $1<$ a Typische Versorgungssequenz von Luxationsfrakturen des Talus. In einem ersten Schritt erfolgt die notfallmäßige geschlossene - und bei Unmöglichkeit offene - Reposition, $\mathbf{b}$ gefolgt von der Anlage eines tibiometatarsalen Fixateur externe. Die definitive Versorgung erfolgt nach Analyse der Computertomographie und entsprechender Planung im Intervall

tenden Instabilitäten, um eine funktionelle Nachbehandlung bei stabilen, nicht gelenkübergreifenden Osteosynthesen zu ermöglichen $[3,19,51]$. Eine offene Reposition und Refixierung von größeren Fragmenten bei peripheren Talusfrakturen ist genauso entscheidend für eine funktionelle Nachbehandlung und für das Outcome der Patienten. Kleine, nichtrekonstruierbare Fragmente können oft nur reseziert werden.

Luxationsfrakturen, die meist als Folge großer Gewalteinwirkung auftreten, sind aufgrund ihrer konsekutiven Weichteilverletzungen mit Notfallcharakter unmittelbar zu reponieren $(\bullet A b b .1)$. Ist eine geschlossene Reposition nicht möglich, muss eine offene Reposition über limitierte Zugänge zur Beseitigung von Interponaten bzw. Lösen von Verhakungen erfolgen $[11,35,57,61]$. Je nach Allgemeinzustand des Patienten und logistischen Voraussetzungen kann auch eine definitive Osteosynthese erfolgen. Andernfalls wird die Fraktur zunächst mit einer temporären tibiometatarsalen Transfixierung im Fixateur externe ruhig gestellt [61]. Eine definitive Versorgung sollte nach Abschwellung der Weichteile und präoperativer Planung im Intervall erfolgen [21]. Offene Talusfrakturen sollten nach Débridement und Lavage - wenn möglich - einer primären operativen Versorgung zugeführt werden. Ist aus patientenbezogenen oder logistischen Gründen eine primär definitive Versorgung nicht möglich, erfolgt auch in diesen Fällen zunächst die Transfixierung mittels Fixateur externe [35].
Eine primäre definitive Osteosynthese aller zentralen Talusfrakturen am Unfalltag oder gar innerhalb weniger Stunden hat hingegen in den mittlerweile hier$\mathrm{zu}$ vorliegenden Studien keinen messbaren Einfluss auf das Ergebnis und die Rate avaskulärer Nekrosen bei Talusfrakturen [4, 22, 41, 51, 54]. Die definitive Versorgung sollte daher von einem erfahrenen Team nach entsprechender Vorbereitung und Planung sowie dem Abschwellen der Weichteile unter optimalen Bedingungen erfolgen [50]. Eine CT sollte nach Grobreposition von Luxationsfrakturen erfolgen, damit eine sinnvolle Analyse und Planung möglich ist.

Nach kritischer Reevaluierung der Weichteilverhältnisse und der Durchblutungssituation sowie der präoperativen CT-Planung erfolgt die definitive Versorgung. In Abhängigkeit der Frakturmorphologie und unter Berücksichtigung der lokalen Anatomie, insbesondere der Gefäßanastomosen im Bereich des Sinus und Canalis tarsi sowie dem Lig. deltoideum, stehen mehrere operative Zugänge und Osteosyntheseverfahren zur Verfügung.

Ob eine Osteosynthese auch bei nicht dislozierten Talushals- und Taluskorpusfrakturen sinnvoll ist, um eine aggressivere frühfunktionelle Nachbehandlung anstreben zu können, ist nicht gesichert [61]. In diesen Fällen können perkutane oder minimalinvasive Schraubenosteosynthesen mit der Verwendung von kanülierten Schrauben, ggf. unter arthroskopischer Kontrolle, zur Anwendung kommen.

Bei allen dislozierten Talushalsfrakturen ist eine anatomische Reposition unter
Sichtkontrolle von medial nach lateral über einen anteromedialen und zusätzlichen lateralen Zugang unverzichtbar [11, 41]. Nach temporärer Kirschner-Drahtfixierung haben sich bei einer Schraubenosteosynthese nahe der Gelenkfläche Doppelgewindeschrauben oder die Verwendung einer Kopfraumfräse zum Versenken des Schraubenkopfes unter Knorpelniveau als vorteilhaft gezeigt. Bei der Schraubenosteosynthese sollte auf die Vermeidung des Zugeffektes geachtet werden, um insbesondere eine Verkürzung der medialen Seite des Talus mit nachfolgender Varusfehlstellung nicht zu provozieren. Bei ausgeprägter medialer Trümmerzone kann zur medialseitigen Stabilisierung und zur Neutralisation des Varusstresses im Talushalsbereich alternativ eine entsprechend anatomisch angepasste winkelstabile Talusplatte eingesetzt werden $[13,41]$.

Um eine ausreichende Übersicht und exakte Gelenkreposition bei dislozierten Taluskorpusfrakturen zu erreichen, ist regelhaft eine zusätzliche Innenknöchelosteotomie erforderlich, falls nicht ohnehin eine Innenknöchelfraktur vorliegt. Zur Überprüfung der Gelenkkongruenz im Subtalargelenk wird wiederum ein lateraler Zugang verwendet. Nach anatomischer Reposition kann bei einfachem Frakturverlauf eine Schraubenosteosynthese in typischer Weise von anteromedial nach posterolateral durchgeführt werden. Bei einer ausgedehnten medialen Trümmerzone sollte vorzugsweise eine Stabilisierung mittels anatomisch angepasster winkelstabiler Platten erfolgen. Ebenfalls 
kann die laterale Frakturkomponente, die durch eine Schraubenosteosynthese nicht ausreichende Stabilität findet, mit einer winkelstabilen Miniplatte versorgt werden (• Abb. 2).

Wenig Aufmerksamkeit wurde in der Literatur bislang den Impressionsfrakturen der medialen Gelenkfacette gewidmet. Wird diese jedoch nicht reponiert, droht ein chronisches Einsinken derselben einer nachfolgenden Varusabweichung des Rückfußes. Diese Impressionen werden über einen tief angelegten medialen Zugang etwas oberhalb des tastbaren Sustentaculum tali dargestellt und angehoben [61]. Hierbei dient die mediale Facette des Kalkaneus als Schablone (- Abb.3). Zur Unterfütterung der Defekte kann autologe Spongiosa von der distalen Tibiametaphyse oder dem Kalkaneus gewonnen werden. Die Stabilisierung erfolgt vorzugsweise mit kleinen, winkelstabilen Platten [41].

Trümmerfrakturen des hinteren Anteils des Taluskorpus, die vorwiegend bei komplexen Fußverletzungen anzutreffen sind, und Frakturen des Proc. posterior tali erfordern einen posterolateralen oder (seltener) posteromedialen Zugang [41]. Für eine ausreichende Übersicht über das obere und untere Sprunggelenk zur anatomischen Gelenkreposition ist der Einsatz eines Femurdistraktors, der zwischen Tibia und Kalkaneus aufgespannt wird, sehr hilfreich. Die Osteosynthese erfolgt vorzugsweise mittels Kleinfragmentschrauben, die in posterior-anteriorer Richtung eingebracht werden (• Abb. 2). Dabei muss eine Irritation der Flexor-hallucislongus-Sehne vermieden werden.

Frakturen des Proc. fibularis tali sind über den anterolateralen Zugang oder schräg verlaufenden Ducroquet-OllierZugang gut zu erreichen. Die subtalare Arthroskopie über anterolaterale und posterolaterale Portale bietet wie bei intraartikulären Kalkaneusfrakturen die Möglichkeit der exakten Kontrolle der geschlossenen Reposition bei einfachen Frakturformen [6]. Nach anatomischer Reposition und temporärer Fixierung mittels 1,0-Kirschner-Draht erfolgt die Osteosynthese mittels Minititanschrauben, die unterhalb der Knorpel-KnochenGrenze eingebracht werden und für eine ausreichende Stabilität sorgen. Kleinere,

Trauma Berufskrankh 2016 · [Suppl 3]: 18:S231-S240 DOI 10.1007/s10039-015-0091-8

๑) Springer-Verlag Berlin Heidelberg 2015

\section{K. Hartwich · S. Rammelt}

\section{Talus- und Kalkaneusfrakturen. Was gibt es Neues?}

\section{Zusammenfassung}

Die Therapie dislozierter Talus- und Kalkaneusfrakturen ist mit einer erheblichen Lernkurve verbunden. Fehlheilungen führen zu signifikanten Einschränkungen der globalen Fußfunktion sowie schmerzhaften posttraumatischen Arthrosen. Während die sofortige Reposition von Luxationen und Luxationsfrakturen eine Notfallindikation darstellt, hat der Zeitpunkt der definitiven Osteosynthese keinen messbaren Einfluss auf das Behandlungsergebnis oder das Auftreten einer avaskulären Nekrose bei zentralen Talusfrakturen. Für die Versorgung dislozierter Talusfrakturen mit zentraler Trümmerzone oder medialer Gelenkimpression stehen neben der klassischen Schraubenosteosynthese anatomisch angepasste winkelstabile Platten zur Verfügung. Die ideale Therapie dislozierter, intraartikulärer Kalkaneusfrakturen wird kontrovers diskutiert. Aufgrund der sehr variablen Frakturanatomie und der vulnerablen Weichteildeckung ist ein individuelles Behandlungskonzept angezeigt. Zur Minimierung von Wundrandnekrosen bei Verwendung des erweitert lateralen Zuganges sind für ausge- wählte Frakturen weniger invasive Osteosynthesen mit Kontrolle der Gelenkreposition über einen Sinus-tarsi-Zugang eine gute Alternative. Hierfür stehen neben Schrauben intramedulläre, verriegelbare Nägel und modifizierte, eingeschobene Platten zur Verfügung. Dislozierte extraartikuläre sowie einfache intraartikuläre Kalkaneusfrakturen können einer perkutanen Osteosynthese zugeführt werden. Unabhängig von der Art der Osteosynthese ist eine frühfunktionelle Nachbehandlung anzustreben. Periphere Frakturen des Talus und Kalkaneus entstehen häufig nach (Sub-)Luxationen im Subtalar- und Chopart-Gelenk. Sie werden nicht selten übersehen und resultieren unbehandelt in schmerzhaften Arthrosen. Ist eine exakt anatomische Reposition dieser Gelenkfrakturen nicht möglich, so ist die Resektion nicht refixierbarer Fragmente indiziert.

Schlüsselwörter Luxation - Osteosynthese - Arthrose . Reposition $\cdot$ Nachbehandlung

\section{Talus and calcaneal fractures. What's new?}

\section{Abstract}

The treatment of displaced fractures of the talus and calcaneus is associated with a considerable learning curve. Malunion results in significant limitations of global foot function and painful posttraumatic arthritis. While early reduction of dislocations and fracture dislocations represent an emergency situation, the timing of definitive fixation has no measurable impact on the results and the incidence of avascular necrosis in central fractures of the talus. For internal fixation of displaced fractures with central comminution or medial joint impaction, anatomically shaped interlocking plates are available in addition to screws. The ideal treatment of displaced intra-articular calcaneal fractures is still controversial. Because of the variable fracture patterns and the vulnerable soft tissue cover, an individual treatment concept is advisable. In order to minimize the wound margin necrosis associated with extensile lateral approaches, selected fractures should be treated with

less invasive fixation while controlling joint reduction via a sinus tarsi approach. Fixation in these cases is achieved with screws, intramedullary locking nails or modified plates that are inserted subcutaneously. Displaced extra-articular and simple intra-articular fractures can be reduced and fixed percutaneously. Functional aftertreatment aims at early rehabilitation independent of the kind of fixation. Peripheral fractures of the talus and calcaneus frequently result from subluxation and dislocation at the subtalar and Chopart's joints. They are still regularly overlooked and result in painful arthritis if left untreated. If an exact anatomical reduction of these intraarticular fractures is impossible, resection of small fragments is indicated.

\section{Keywords}

Osteosynthesis · Arthritis · Reduction .

Dislocation · Aftertreatment

nicht anatomisch refixierbare Fragmente, oder Fragmente mit starker Knorpelkontusion werden vorzugsweise exzidiert. Gleiches gilt für Frakturen des Proc. pos- terior tali zur Vermeidung einer posttraumatischen Arthrose [15].

Prinzipiell sollte das Behandlungskonzept so strukturiert und umsetzbar sein, 

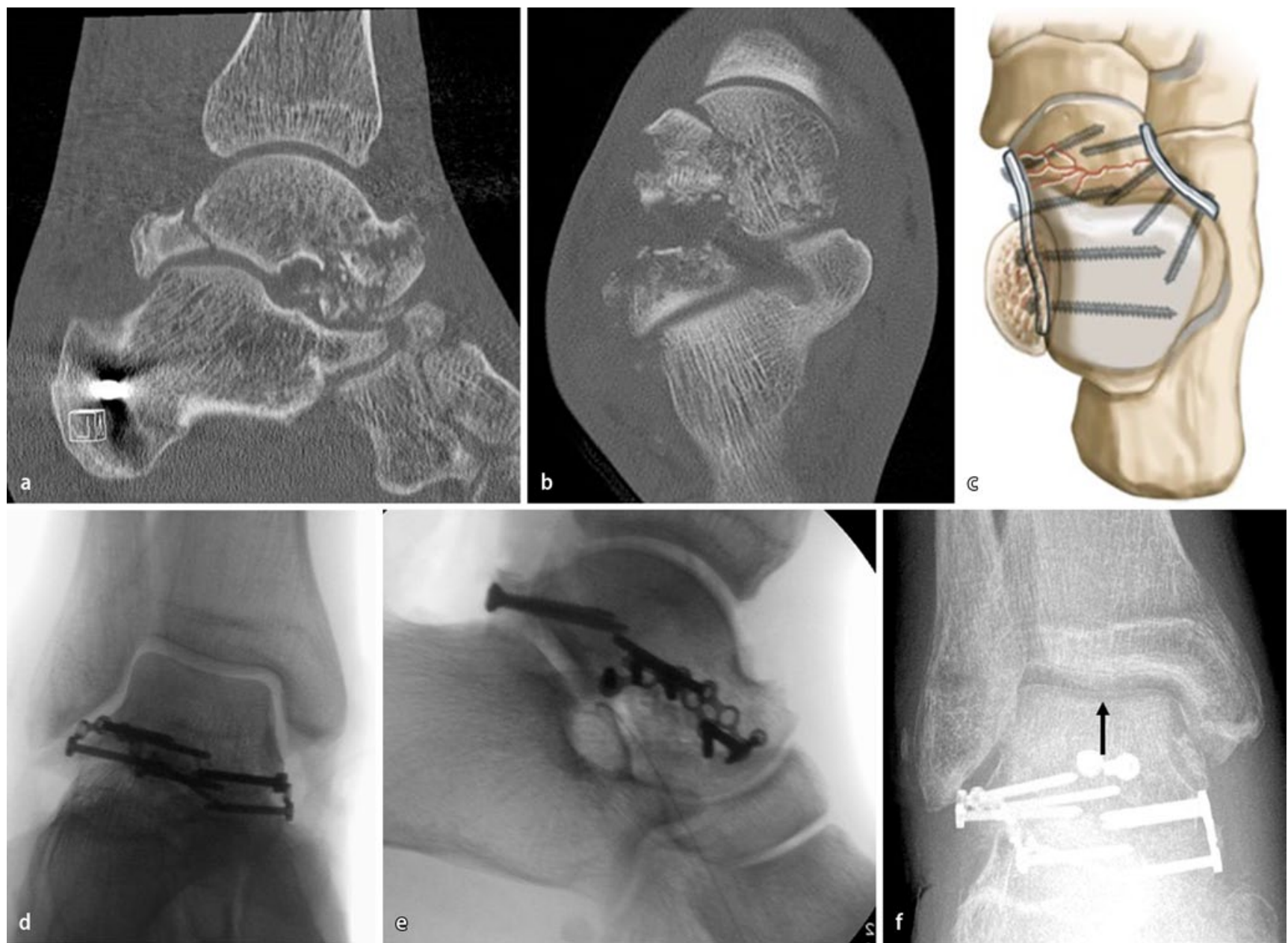

Abb. $2 \Delta$ a, b Die Computertomographie desselben Patienten wie in - Abb. 1 zeigt eine typische zentral gelegene Trümmerzone und ein kleines, ebenfalls frakturiertes Taluskopffragment. c-e In diesem Fall ist die bilaterale Plattenosteosynthese mit Überbrückung des Defektes eine gute Therapiealternative zur klassischen Schraubenosteosynthese. Der frakturierte Proc. posterior wurde zusätzlich mittels Schraubenosteosynthese versorgt. f Nach 8 Wochen ist eine Zone vermehrter Strahlentransparenz (Pfeil) im Talusdom zu sehen (positives Hawkins-Zeichen als sicherer Hinweis für eine Remodellierung des Knochens)

dass bei einer übungsstabilen Osteosynthese eine frühfunktionelle Behandlung begonnen werden kann. Die in bis zu $100 \%$ erkennbaren radiologischen Anzeichen einer posttraumatischen Arthrose korrelieren nicht immer mit dem klinisch funktionellen Ergebnis.

Zahlreiche Studien haben mittlerweile eine Korrelation zwischen dem initialen Dislokationsgrad bei den häufigen Talushalsfrakturen und dem Auftreten einer avaskulären Nekrose nachgewiesen (Übersicht in [61]). Vallier et al. [54] haben in einer Subgruppenanalyse festgestellt, dass auch innerhalb der Gruppe der Hawkins-Typ-II-Frakturen die Luxation im Subtalargelenk einen wichtigen Faktor für die Entstehung einer avaskulären Nekrose darstellt. Sie bezeichneten diese
Verletzungen daher als Hawkins Typ IIB. Entscheidend für die Prognose ist jedoch, ob es sich um eine partielle Nekrose handelt, wie sie in der Magnetresonanztomographie (MRT) oder Gewebsproben fast immer nachweisbar ist, oder eine totale Nekrose des Taluskorpus, die zu einem Kollaps des Talusdomes führt [41]. Für Letztere bestehen keine Notwendigkeit einer prolongierten Entlastung und keine schlechtere Prognose [53, 54]. Es lohnen sich in diesen Fällen sogar die sekundäre Osteotomie und anatomische Rekonstruktion von fehlverheilten Talusfrakturen oder Pseudarthrosen [61]. Das Auftreten eines Hawkins-Zeichens 6 bis 10 Wochen nach der Fraktur, also einer weniger röntgendichten subchondralen Zone im Talusdom (- Abb. 2), ist ein sicherer Hin- weis auf eine Remodellierung des Talus und somit das Ausbleiben einer Totalnekrose des Taluskorpus mit nachfolgendem Kollaps [52, 61].

\section{Kalkaneusfrakturen}

\section{Vorbemerkungen}

Dislozierte intraartikuläre Kalkaneusfrakturen zählen zu den größeren Herausforderungen für den behandelnden Chirurgen mit einer erheblichen Lernkurve und zu den kontrovers diskutierten Themen [30, 34, 45]. Die Behandlungsempfehlungen reichen von der klassischen operativen Versorgung mittels Plattenosteosynthese über einen ausgedehnten lateralen Zugang über weniger invasive Verfahren 

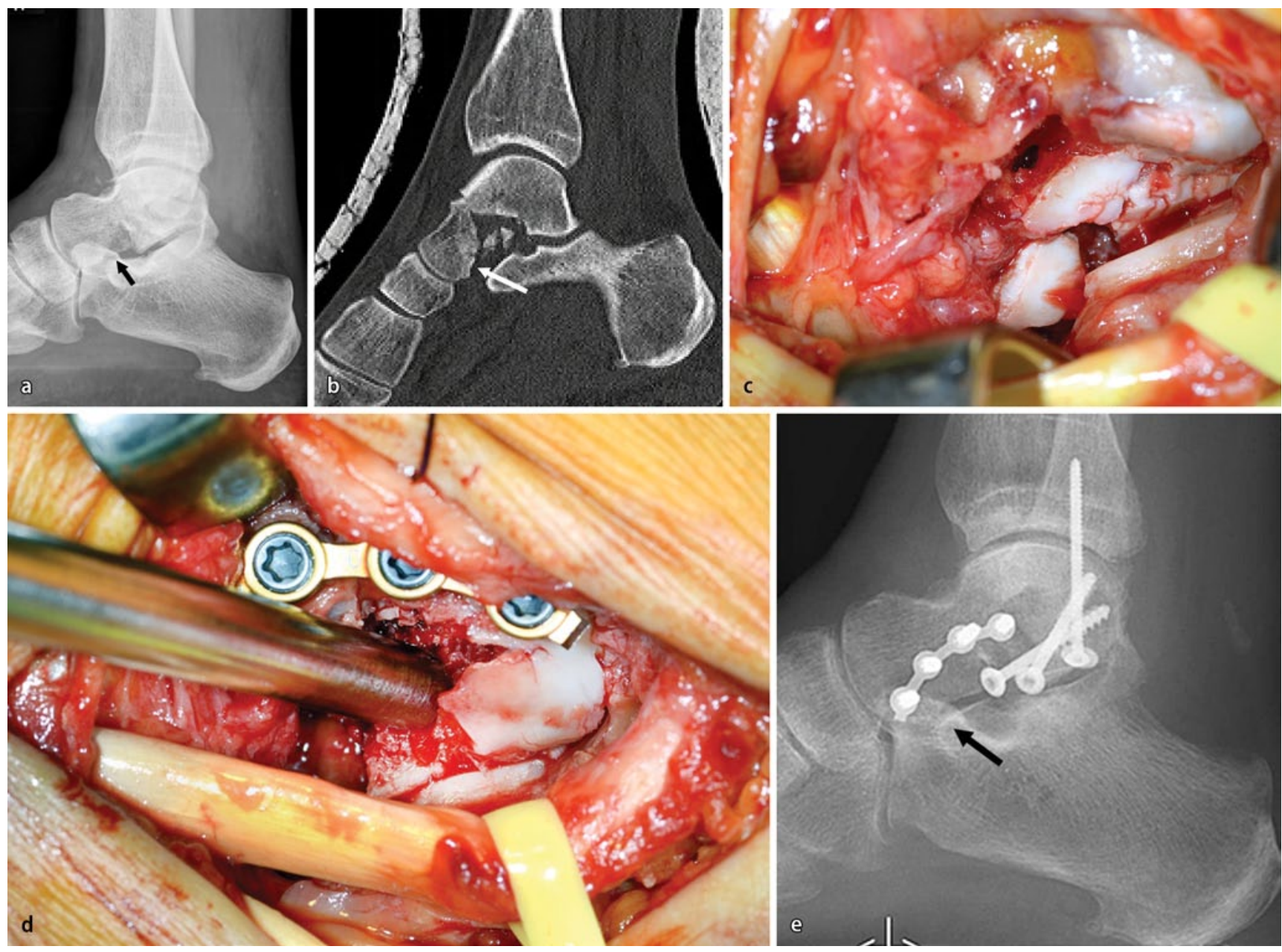

Abb. $3 \Delta$ a, b Versorgung einer Impressionsfraktur der medialen Gelenkfacette des Talus (Pfeile) mittels c, d Aufstößelung, lokaler Spongiosaplastik und medialer Plattenosteosynthese. e Die postoperative Aufnahme zeigt die Wiederherstellung der medialen Gelenkkongruenz (Pfeil). (Abb. mod. nach [41])

wie eingeschobene Platten oder Kalkaneusnagel bis hin zur perkutanen Osteosynthese und rein konservativen Therapie. Mittlerweile scheint sich die Erkenntnis durchzusetzen, dass keine einzelne Behandlungsmethode für alle Verletzungsmuster geeignet ist [32].

Aufgrund des typischen Unfallmechanismus mit axialer Gewalteinwirkung im Rahmen von Stürzen aus der Höhe oder Dezelerationstraumata sind Kalkaneusfrakturen zu mehr als drei Viertel intraartikuläre Frakturen [60]. Davon haben wiederum $97 \%$ eine intraartikuläre Verwerfung der posterioren Gelenkfacette, $59 \%$ eine zusätzliche Beteiligung des Kalkaneokuboidgelenks und $8 \%$ eine Involvierung der anterioren Gelenkfacette [57].

Erst anhand einer CT lässt sich das genaue Ausmaß der Verletzung erkennen.
Daher ist nach Sicherung der Diagnose in den Standardröntgenaufnahmen eine CT für die genaue Analyse der Frakturmorphologie inklusive der Klassifikation sowie zur Operationsplanung unabdingbar. Zwipp et al. [57] konnten in einer Analyse von über 200 Frakturen in $58 \%$ der Fälle 5 relativ konstante Hauptfragmente nachweisen. Neben der Anzahl der Hauptfragmente werden die Beteiligung der Gelenkfacetten (maximal 3), der Schweregrad der Weichteilverletzung nach Tscherne und Oestern und evtl. regionale Zusatzfrakturen in einer 12-Punkte-Frakturskala erfasst. Die international am häufigsten angewendete Frakturklassifikation ist diejenige von Sanders [44], die anhand der koronaren CT-Schnitte auf der Anzahl der Frakturlinien in der posterioren Gelenkfacette basiert.
- Typ I: undisloziert,

- Typ II: 1 Frakturlinie,

- Typ III: 2 Frakturlinien,

- Typ IV: 3 und mehr Frakturlinien.

Die AO/ICI-Klassifikation (Integral Classification of Injuries der Arbeitsgemeinschaft für Osteosynthesefragen) unterteilt die Kalkaneusfrakturen nach dem bekannten ABC-Prinzip in extraartikulär (Typ A), intraartikulär (Typ B), Luxationsfrakturen (Typ C) sowie in reine Luxationen (Typ D). Zusätzlich erfolgt die detaillierte Beschreibung der Frakturschwere in Untergruppen [58].

\section{Versorgungsstrategie und neue Entwicklungen}

Wie bei den Talusfrakturen hat auch bei den Kalkaneusfrakturen eine exakte ana- 

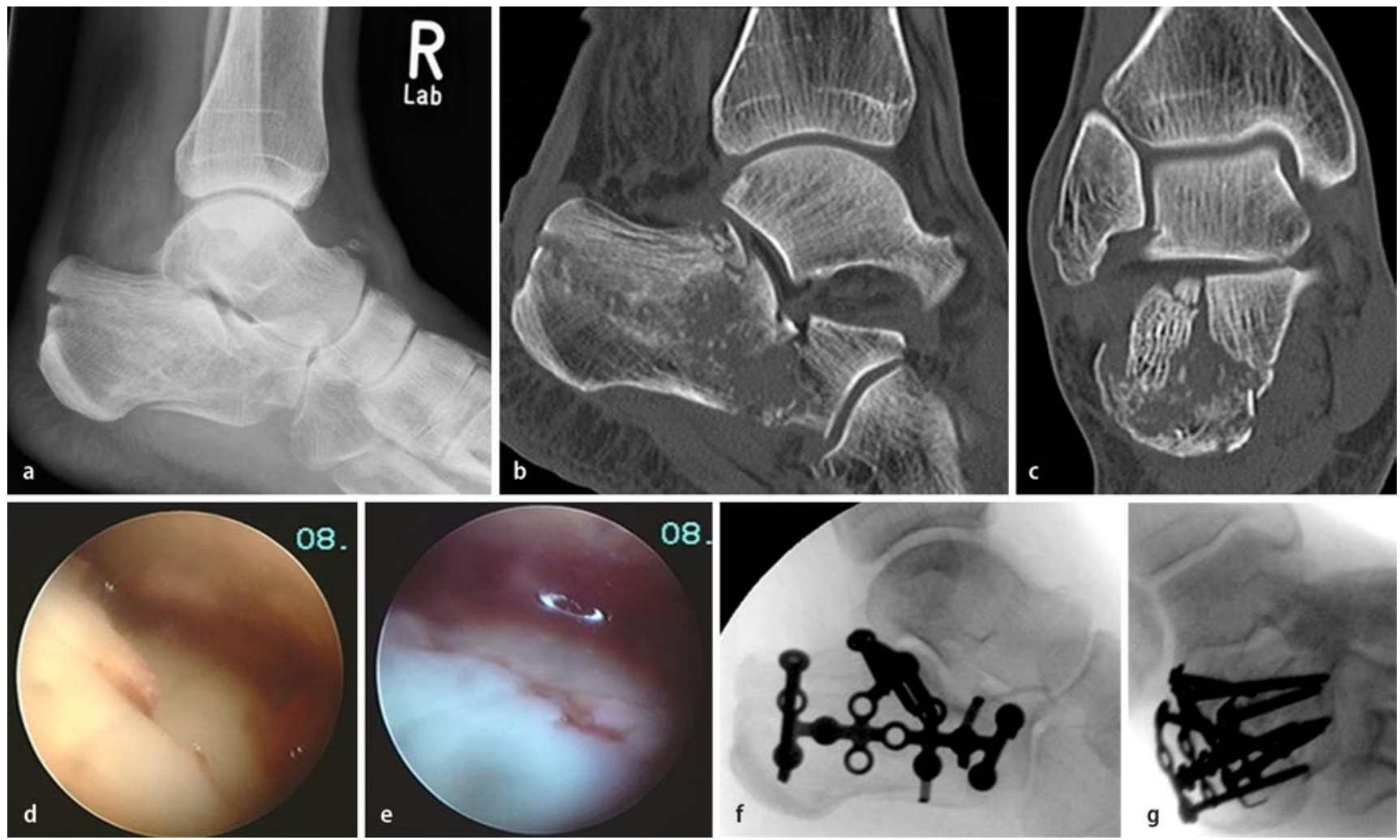

Abb. 4 A a-c Klassische laterale Plattenosteosynthese bei dislozierter, intraartikulärer Kalkaneusfraktur vom Typ Sanders II mit d-e einem winkelstabilen Implantat. $\mathbf{f}-\mathbf{g}$ Die anatomische Korrektur der Gelenkfläche kann mit intraoperativer offener Arthroskopie oder einer 3D-Bildgebung exakt kontrolliert werden

tomische Wiederherstellung der korrekten Achsverhältnisse und Gelenkkongruenz am Rückfuß zur Vermeidung von posttraumatischen Arthrosen, Fehlstellungen des gesamten Fußes sowie Weichteilimpingement oberste Priorität.

Aufgrund der prekären und vulnerablen Weichteilbedeckung des Fersenbeines kommt einem individuell abgestimmten Weichteilmanagement eine Schlüsselrolle bei der Vermeidung von Komplikationen zu. Eine notfallmäßige Operationsindikation besteht bei offenen Frakturen, akut assoziiertem Kompartmentsyndrom und der drohenden Hautperforation aufgrund des Fragmentdruckes stark dislozierter Frakturen. Hier sind in erster Linie die sog. Entenschnabelfrakturen mit weit nach kranial disloziertem Oberrand des Tuber calcanei zu nennen $[14,61]$. Eine frühe, definitive Weichteildeckung dient der Infektprophylaxe und frühen funktionellen Nachbehandlung bei verbleibenden Weichteildefekten nach offenen Frakturen [7, 24].

Aufgrund klinischer und biomechanischer Studien wird im Allgemeinen die
Operationsindikation bei dislozierten intraartikulären Kalkaneusfrakturen mit einer Gelenkstufe von $2 \mathrm{~mm}$ und relevanter extraartikulärer Deformität (Verbreiterung, Varus, Valgus) gestellt. Die Osteosynthese erfolgt klassischerweise mittels Plattenosteosynthese über einen ausgedehnten lateralen Zugang. Hierzu stehen zahlreiche, den anatomischen Gegebenheiten angepasste, z. T. winkelstabile Implantate zur Verfügung (• Abb. 4). Unabhängig vom gewählten Plattendesign muss darauf geachtet werden, dass nach anatomischer Rekonstruktion der Gelenkflächen von medial nach lateral eine stabile Verankerung des Sustentaculum, Tuber und Proc. anterior zur Fixierung der wiederhergestellten Anatomie erfolgt. Bei Tongue-type-Frakturen, die weit in die Gelenkfläche auslaufen, kann die Reposition der posterioren Gelenkfacette erschwert sein, insbesondere, wenn die Fraktur schon mehrere Tage zurückliegt. Für diese Fälle hat Sanders [46] eine Osteotomie dieses Fragmentes hinter der Gelenkfacette entwickelt, welche die anatomische Reposition der Gelenkfläche er- leichtert, indem sozusagen eine Joint-depression-type-Fraktur erzeugt wird. Die sichere Kontrolle der anatomischen Reposition der Gelenkfacetten erfolgt im Zweifelsfall mittels intraoperativer Arthroskopie oder 3-dimensionaler Bildgebung $[37,43]$.

Die relativ hohe Rate an Wundrandnekrosen bei Verwendung des ausgedehnt lateralen Zuganges hat zur Suche nach alternativen Zugangswegen geführt. Während der direkte laterale Zugang nach $\mathrm{Pal}$ mer keine Vorteile zu erbringen scheint, lassen sich Wundprobleme mit einem kleinen Zugang über dem Sinus tarsi deutlich reduzieren (• Abb. 5). Dieser Zugang erlaubt eine direkte Einsicht auf die posteriore Gelenkfacette und direkte Manipulation der Gelenkfragmente, während die übrigen Hauptfragmente perkutan reponiert und fixiert werden [27]. Diese Technik ist für weniger komplexe Frakturformen geeignet $[32,49]$. Für die Osteosynthese stehen neben Schrauben mittlerweile auch modifizierte Platten und intramedulläre Nägel zur Verfügung, die insbesondere bei schlechterer Knochenqua- 

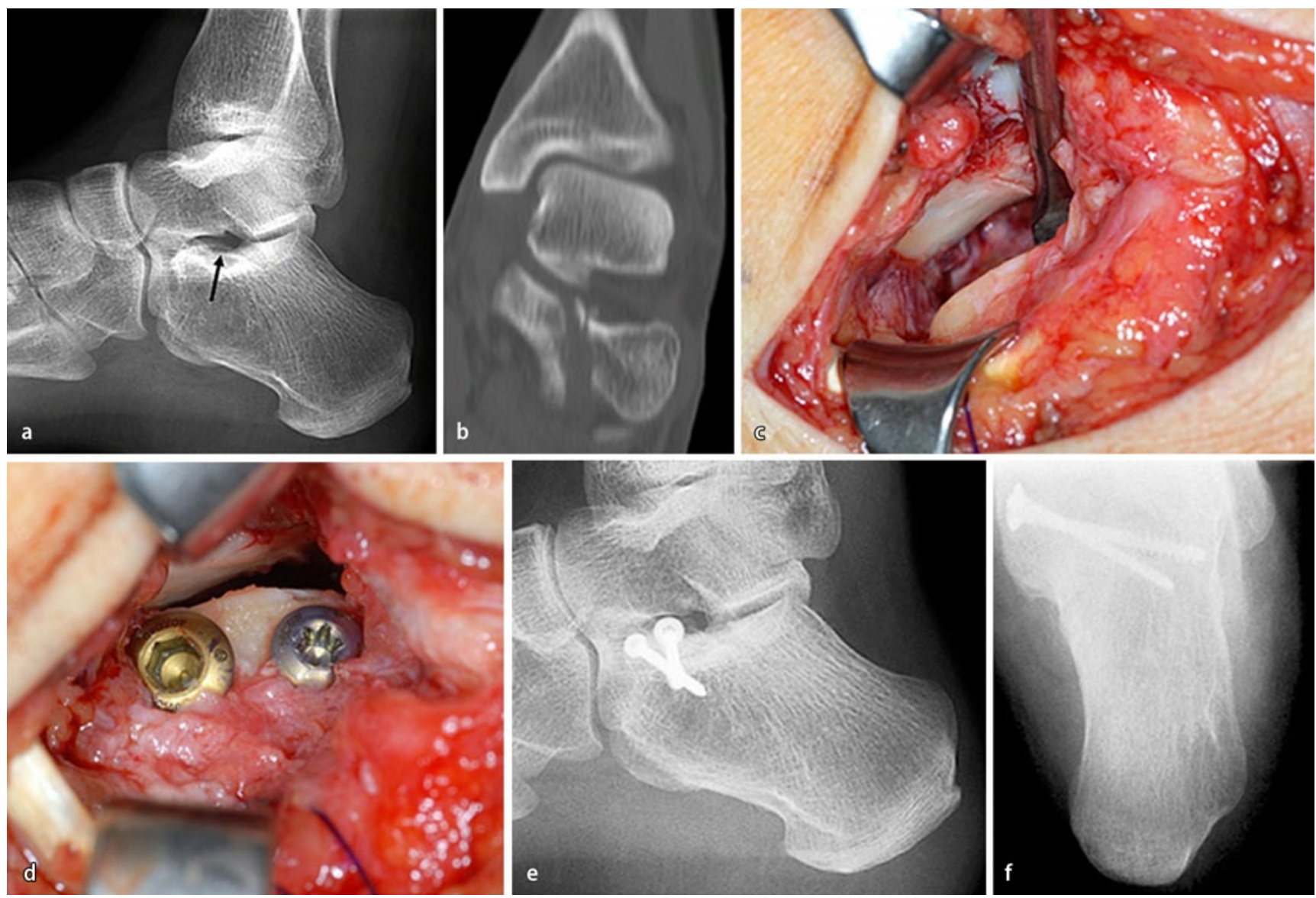

Abb. $5 \Delta$ Schraubenosteosynthese einer isolierten Fraktur des Sustentaculum tali (a, b) über einen direkten medialen Zugang (c). Die Kongruenz der medialen Gelenkfacette wird so wiederhergestellt (d-f) und einer Varusdeformität sowie einer posttraumatischen Arthrose vorgebeugt. (Abb. mod. nach [12])

lität eine winkelstabile Verankerung der Hauptfragmente mittels über ein Zielgerät gesetzten Verriegelungsbolzen erlauben $[16,60]$.

Dislozierte extraartikuläre Frakturen (Typ A, Sanders I und IIC) sowie einfache intraartikuläre Frakturen (Typ B, Sanders IIA und IIB) können mit sehr guter Prognose mittels rein perkutaner Repositionstechnik und bei Gelenkbeteiligung unter arthroskopischer Sicht einer perkutanen Schraubenosteosynthese zugeführt werden $[26,37,39,40,50,55]$. Eine Osteosynthese mit Kirschner-Drähten ist hingegen mit dem Risiko einer sekundären Dislokation behaftet. Überstehende oder gelenkübergreifende Kirschner-Drähte sollten - wenn möglich - vermieden werden [38].

Wichtig sind bei allen weniger invasiven und perkutanen Osteosynthesen die sorgfältige präoperative Analyse und Kontrolle der exakten Reposition der Gelenkfläche mittels Arthroskopie oder 3D-
Bildwandler, um nicht die Weichteilschonung mit einem erhöhten Arthroserisiko zu erkaufen [39, 43]. Perkutane Repositionstechniken und Osteosynthesen kommen als temporäre oder definitive Verfahren auch bei vulnerablen, kritischen Weichteilverhältnissen v. a. bei Patienten mit einem erhöhten perioperativen Risikoprofil als Behandlungsalternative in Betracht.

Atypische, peripher gelegene Fakturen des Kalkaneus werden nicht selten übersehen und können zu erheblichen Beschwerden führen. Sie entstehen häufig durch Luxationsmechanismen im Subtalargelenk (am Sustentaculum tali) bzw. im Chopart-Gelenk (am Oberrand des Proc. anterior calcanei). Bei isolierten dislozierten Frakturen des Sustentaculum tali mit Gelenkstufe oder Verwerfung der medialen Gelenkfacette des Subtalargelenks ist eine anatomische Rekonstruktion über einen direkten, gewebeschonenden medialen Zugang indiziert.
Nach Retraktion der Sehnen und Reposition der Fraktur erfolgt die Osteosynthese mittels Kleinfragmentschrauben von medial nach lateral und leicht nach plantar und posterior gerichtet (• Abb. 6). Bei der mit 18 Patienten bisher größten Untersuchungsreihe in der Literatur zeigt sich diese Technik v. a. bei isolierten Sustentaculumfrakturen hinsichtlich der Vermeidung von Komplikationen und eines guten funktionellen Ergebnisses vielversprechend [12].

Bei allen nicht gelenkübergreifenden Osteosynthesen kann auf eine zusätzliche Protektion verzichtet und eine frühfunktionelle Nachbehandlung im patienteneigenen Schuh angestrebt werden. Nach allen Osteosyntheseverfahren erfolgt eine Teilbelastung über 6 bis 12 Wochen je nach Frakturmorphologie und Knochenqualität. Trotz anatomischer Reposition verbleibt das Risiko einer posttraumatischen Arthrose schon aufgrund des primären Knorpelschadens im Rah- 

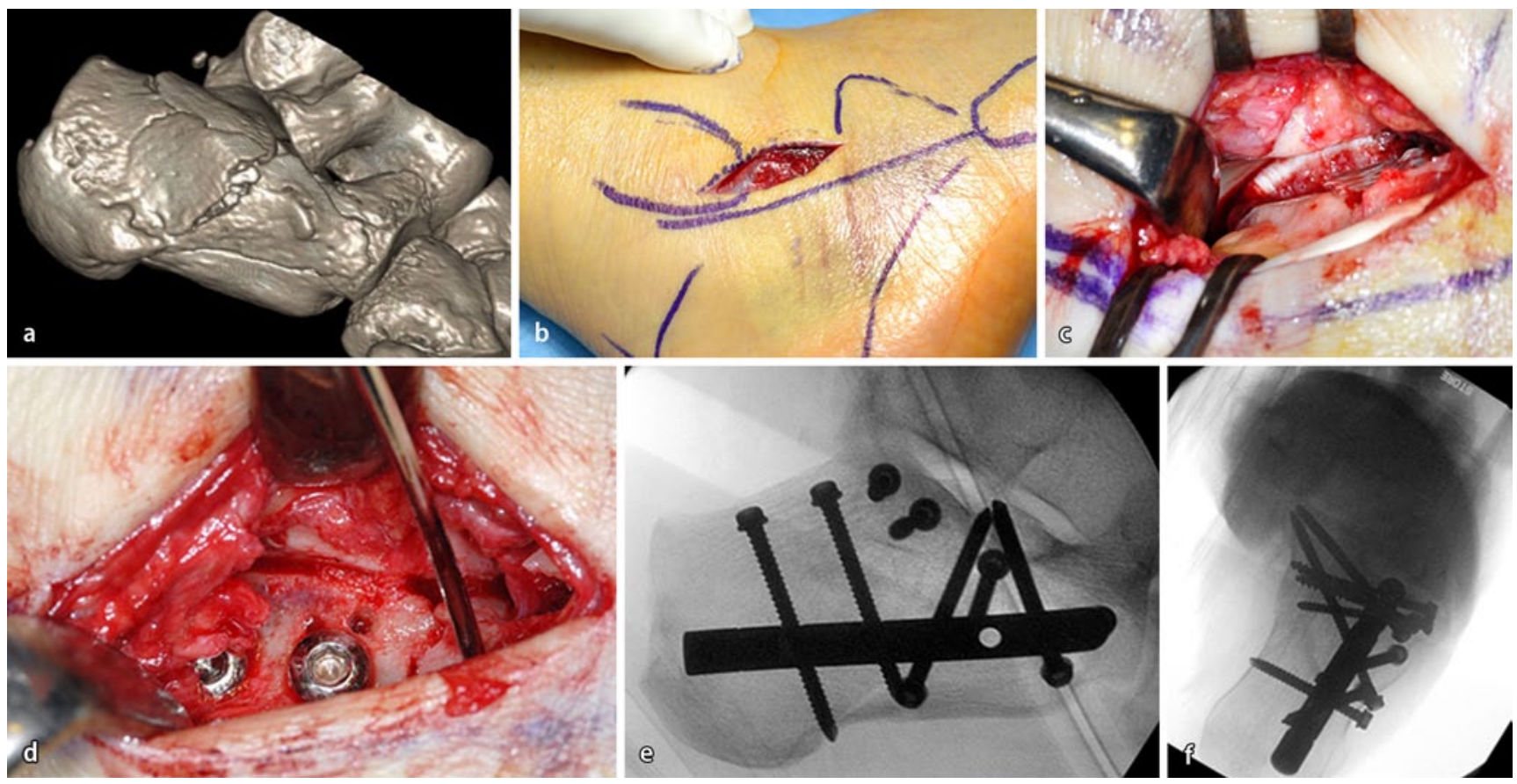

Abb. $6 \Delta$ a-c Für einfachere Frakturformen lässt sich eine ausreichende Gelenkübersicht und anatomische Reposition oft über einen kleinen Zugang direkt über dem Sinus tarsi erzielen. d-f Die Osteosynthese erfolgt mittels Schrauben oder kleinen, eingeschobenen Platten. Alternativ kann - insbesondere bei osteoporotischem Knochen - ein intramedulläres Implantat (im Beispiel: C-Nail) sinnvoll sein. (Abb. mod. nach [60])
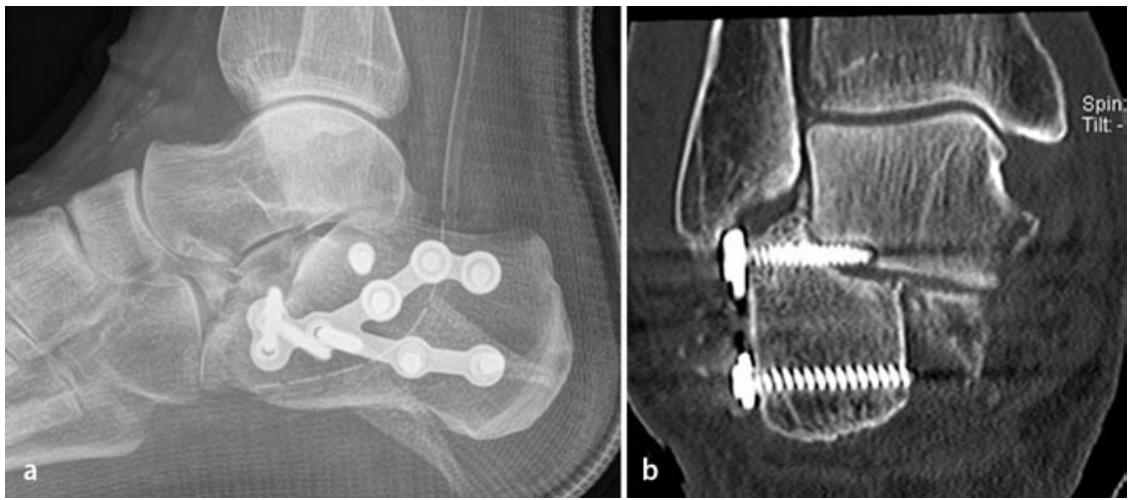

Abb. $7 \Delta$ a, b Persistierende Fehlstellung nach Plattenosteosynthese einer dislozierten, intraartikulären Kalkaneusfraktur über einen ausgedehnt lateralen Zugang. Ein solches Vorgehen vereinigt die Nachteile der operativen und konservativen Therapie

men des axialen Stauchungstraumas. Bei relevanter Bewegungseinschränkung und prominentem Osteosynthesematerial besteht die Möglichkeit einer Metallentfernung mit direkter arthroskopischer Evaluierung und Arthrolyse des Subtalargelenks sowie Tenolyse der Peronealsehnen zur Verbesserung des funktionellen Ergebnisses [37]. Bei symptomatischer Arthrose ist die In-situ-Arthrodese des Subtalargelenks indiziert, die technisch einfacher und mit besseren Ergebnissen assoziiert ist als eine Korrekturarthrodese nach fehlverheilter Kalkaneusfraktur [31]. Eine primäre Arthrodese, wie verschiedentlich für Sanders-Typ-IV-Frakturen empfohlen, hat in einer prospektiv randomisierten Studie hingegen keine Vorteile gegenüber der Osteosynthese erbracht [9]. In dieser Studie war lediglich bei einem Patienten der Osteosynthesegruppe eine sekundäre Arthrodese notwendig. Diese niedrige Inzidenz deckt sich auch mit den eigenen Erfahrungen [42].

In den vergangenen Jahren wurde mit aufwendigen prospektiv randomisierten
Studien versucht, evidenzbasierte Empfehlungen für die operative oder konservative Behandlung von Kalkaneusfrakturen auszusprechen $[1,8,17]$. Wenngleich sich durchaus praxisrelevante Erkenntnisse aus diesen Studien gewinnen lassen, zeigen sie doch auch die Grenzen dieser Methodik auf unfallchirurgischem Gebiet [56]: relativ geringe Rekrutierungszahlen über einen großen Zeitraum, daraus folgend eine oft geringe Zahl von Eingriffen pro Operateur sowie relativ wenig Patienten pro Subgruppe bei detaillierter Analyse der sehr variablen Frakturen, was die statistische Auswertung erschwert ( $\alpha-\mathrm{Fe}$ hler).

Eine kürzlich erschienene Studie von Griffin et al. [17] fand keinen signifikanten Unterschied bezüglich der funktionellen Ergebnisse durchschnittlich 2 Jahre nach operativer bzw. konservativer Therapie dislozierter, intraartikulärer Kalkaneusfrakturen vom Typ Sanders II-III. Allerdings konnten in diese Studie nur 151 von 502 Patienten rekrutiert werden, was eine gewisse Selektion weniger schwer dislozierter Frakturen vermuten lässt. Im Schnitt wurden von jedem beteiligten Operateur nur 2 Frakturen pro Jahr versorgt, was bei einer Verletzung 
mit einer sehr flachen Lernkurve ein gewichtiger Nachteil bezüglich der möglichen Komplikationen ist [30]. In $22 \%$ der Fälle verblieb eine Gelenkstufe von mehr als $2 \mathrm{~mm}$, was eine signifikante Lastumverteilung mit einem erheblichen Arthroserisiko bedeutet $[25,48]$. Eine operative Therapie, die keine anatomische Reposition ergibt, vereinigt jedoch die Nachteile des operativen und konservativen Vorgehens (• Abb. 7).

Die prospektiv randomisierte Studie von Ågren et al. [1] ergab einen Trend $\mathrm{zu}$ höherer Lebensqualität sowie weniger Schmerzen nach operativer Therapie gegenüber konservativer Therapie bei einer Nachuntersuchungsdauer von 8 bis 12 Jahren. Allerdings wurden nur 1 bis 2 Frakturen pro Operateur pro Jahr versorgt, und in $40 \%$ verbleib eine Gelenkstufe von mehr als $2 \mathrm{~mm}$, in $10 \%$ sogar über $5 \mathrm{~mm}$. Eine Post-hoc-Analyse derselben Gruppe [2] ergab bei der Aufteilung der Patienten in solche mit guten und schlechten Ergebnissen signifikant mehr Patienten in der ersten Gruppe nach operativer Therapie und anatomischer Reposition. Dies deckt sich mit den Ergebnissen von Buckley et al. [8]. Diese Autoren fanden gemessen an der Lebensqualität (SF-36) und einer visuellen Analogskala im Gesamtkollektiv von über 400 Patienten nur leichte, nichtsignifikante Vorteile der operativen Therapie (laterale Plattenosteosynthese), allerdings waren nach Osteosynthese signifikant weniger sekundäre subtalare Arthrodesen erforderlich. Bei Nichtbetrachtung der Arbeitsunfälle wiesen die operativ behandelten Patienten ein signifikant besseres Ergebnis auf. In dieser Patientengruppe profitierten insbesondere Patienten unter 29 Jahren, mit einem relativ flachen individuellen Böhler-Winkel und höhergradigen Frakturen sowie diejenigen mit anatomischer Reposition bzw. einer Gelenkstufe von weniger als $2 \mathrm{~mm}$ von der Operation.

\section{Fazit für die Praxis}

- Die korrekte anatomische Ausrichtung von Talus und Kalkaneus sowie ihren Gelenken ist essenzielle Voraussetzung für eine normale Funktion des gesamten Fußes. Die Therapie dislozierter Talus- und Kalkaneusfrakturen gehört zu den großen Herausforderungen in der Unfallchirurgie, die Osteosynthese ist mit einer erheblichen Lernkurve verbunden, und fehlverheilte Frakturen führen zu relevanten Einschränkungen der globalen Fußfunktion sowie schmerzhaften posttraumatischen Arthrosen.

- Während die sofortige Reposition von Luxationen und Luxationsfrakturen eine Notfallindikation darstellt, hat der Zeitpunkt der definitiven Osteosynthese keinen messbaren Einfluss auf das funktionelle Behandlungsergebnis oder das Auftreten einer avaskulären Nekrose bei zentralen Talusfrakturen. Das Auftreten eines Hawkins-Zeichens 6 bis 10 Wochen nach der Fraktur ist ein sicherer Hinweis auf eine Remodellierung des Talus und somit das Ausbleiben einer Totalnekrose des Taluskorpus mit nachfolgendem Kollaps.

- Für die Versorgung dislozierter Taluskörper- und -halsfrakturen mit zentraler Trümmerzone oder medialer Gelenkimpression stehen neben der klassischen Schraubenosteosynthese anatomisch angepasste winkelstabile Platten zur Verfügung.

- Periphere Talusfrakturen entstehen häufig im Rahmen subtalarer Luxationen und erfordern entweder die anatomische Reposition oder Resektion kleiner, nichtrefixierbarer Fragmente.

- Die ideale Therapie dislozierter, intraartikulärer Kalkaneusfrakturen wird in der Literatur kontrovers diskutiert. Auch jüngere, prospektiv randomisierte Studien geben aufgrund inhärenter methodischer Probleme keine eindeutige Antwort. Aufgrund der sehr variablen Frakturanatomie und der vulnerablen Weichteildeckung ist ein individuelles Behandlungskonzept angezeigt.

- Zur Minimierung von Wundheilungsstörungen und Wundrandnekrosen bei Verwendung des erweitert lateralen Zuganges sind für ausgewählte Frakturen weniger invasive Osteosynthesen mit Kontrolle der anatomischen Gelenkreposition über einen direkten Sinus-tarsi-Zugang eine gu- te Behandlungsalternative. Für die Osteosynthese stehen neben Schrauben intramedulläre, verriegelbare Nägel und modifizierte, eingeschobene Platten zur Verfügung.

- Dislozierte extraartikuläre (Typ A, Sanders I und IIC) sowie einfache intraartikuläre (Typ B, Sanders IIA und IIB) Kalkaneusfrakturen können mittels perkutaner Repositionstechnik und bei Gelenkbeteiligung unter arthroskopischer Sicht einer perkutanen Schraubenosteosynthese zugeführt werden.

- Isolierte dislozierte Frakturen des Sustentaculum tali mit Gelenkstufe oder Verwerfung der medialen Gelenkfacette des Subtalargelenks sind über einen direkten medialen Zugang erfolgreich mittels Kleinfragmentschrauben zu versorgen.

- Unabhängig von der Art der Osteosynthese ist eine frühfunktionelle Nachbehandlung mit Bewegungsübungen und Teilbelastung des betroffenen Fußes bis zur Konsolidierung der Fraktur anzustreben, wenn die Weichteilverhältnisse dies zulassen.

\section{Korrespondenzadresse}

Prof. Dr. med. S. Rammelt

UniversitätsCentrum für Orthopädie und Unfallchirurgie, Universitätsklinikum Carl Gustav Carus der TU Dresden

Fetscherstr. 74, 01307 Dresden

stefan.rammelt@uniklinikum-dresden.de

\section{Einhaltung ethischer Richtlinien}

Interessenkonflikt. S. Rammelt ist Mitglied der AO Foot \& Ankle Expert Group und erhielt Vortragshonorar von der Firma DePuy Synthes. K. Hartwich gibt an, dass kein Interessenkonflikt besteht.

Dieser Beitrag beinhaltet keine Studien an Menschen oder Tieren.

The supplement containing this article is not sponsored by industry.

\section{Literatur}

1. Ågren PH, Wretenberg P, Sayed-Noor AS (2013) Operative versus nonoperative treatment of displaced intra-articular calcaneal fractures: a prospective, randomized, controlled multicenter trial. J Bone Joint Surg Am 95:1351-1357 
2. Ågren PH, Mukka S, Tullberg T, Wretenberg P, Sayed-Noor AS (2014) Factors affecting long-term treatment results of displaced intra-articular calcaneal fractures. A post-hoc analysis of a prospective, randomized, controlled multicenter trial. J Orthop Trauma 28:564-568

3. Baumhauer JF, Alvarez RG (1995) Controversies in treating talus fractures. Orthop Clin North Am 26:335-351

4. Bellamy JL, Keeling JJ, Wenke J, Hsu JR (2011) Does a longer delay in fixation of talus fractures cause osteonecrosis? J Surg Orthop Adv 20:34-37

5. Bibbo C, Anderson RB, Davis WH (2003) Injury characteristics and the clinical outcome of subtalar dislocations: a clinical and radiographic analysis of 25 cases. Foot Ankle Int 24(2):158-163

6. Boack DH, Manegold S, Haas NP (2004) Therapiestrategie bei Talusfrakturen. Unfallchirurg 107:499-514

7. Brenner P, Rammelt S, Gavlik JM, Zwipp H (2001) Early soft tissue coverage after complex foot trauma. World J Surg 25:603-609

8. Buckley R, Tough S, McCormack R et al (2002) Operative compared with nonoperative treatment of displaced intra- articular calcaneal fractures: a prospective, randomized, controlled multicenter trial. J Bone Joint Surg Am 84-A:1733-1744

9. Buckley R, Leighton R, Sanders D, Poon J, Coles CP, Stephen D, Paolucci EO (2014) Injury characteristics and the clinical outcome of subtalar dislocations: a clinical and radiographic analysis of $25 \mathrm{ca}-$ ses. J Orthop Trauma 28(10):577-583

10. Canale ST, Kelly FB Jr (1978) Fractures oft he neck oft the talus. J Bone Joint Surg (Am) 60:143-156

11. Cronier P, Talha A, Massin P (2004) Central talar fractures-therapeutic consinderations. Injury 35(Suppl 2):SB10-SB22

12. Dürr C, Zwipp H, Rammelt S (2013) Fractures of the sustentaculum tali. Operat Orthop Traumatol 25(6):569-578

13. Fleuriau Chateau PB, Brokaw DS, Jelen BA, Scheid DK, Weber TG (2002) Plate fixation of talar neck fractures: preliminary review of a new technique in twenty-three patients. J Orthop Trauma 16:213219

14. Gardner MJ, Nork SE, Barei DP, Kramer PA, Sangeorzan BJ, Benirschke SK (2008) Secondary soft tissue compromise in tongue-type calcaneus fractures. J Orthop Trauma 22:439-445

15. Giuffrida AY, Lin SS, Abidi N, Berberian W, Berkman A, Behrens FF (2003) Pseudo os trigonum sign: missed posteromedial talar facet fractures. Foot Ankle int 24:642-649

16. Goldzak M, Mittlmeier T, Simon P (2012) Locked nailing for the treatment of displaced articular fractures of the calcaneus: description of a new procedure with calcanail(R). Eur J Orthop Surg Traumatol 22(4):345-349

17. Griffin D, Parsons N, Shaw E, Kulikov N, Hutchinson C, Thorogood M (2014) Operative versus non-operative treatment for closed, displaced, intra-articular fractures of the calcaneus: randomised controlled trial. BMJ 349:94483

18. Hawkins LG (1970) Fractures oft he neck oft the talus. J Bone Joint Surg Am 52:991-1002

19. Kundel K, Braun W, Scherer A (1995) Spätergebnisse nach zentralen Talusfrakturen. Unfallchirurg 98:124-129

20. Kuner EH, Lindenaier HL (1983) Zur Behandlung der Talusfraktur. Kontrollstudie von 262 Behandlungsfällen. Unfallchirurgie 9(35):40

21. Lenz M, Marintschev I, Klos K, Hofmann GO (2014) Therapiestrategien bei Talusfrakturen. Trauma Berufkranh 16:167-172
22. Lindvall E, Haidukewych G, DiPasquale T, Herscovici D Jr, Sanders R (2004) Open reduction and stable fixation of isolated, displaced talar neck and body fractures. J Bone Joint Surg Am 86-A:2229-2234

23. Marti R (1974) Talus und Calcaneusfrakturen. In: Weber BG, Brunner C, Freuler F (Hrsg) Die Frakturenbehandlung bei Kindern und Jugendlichen. Berlin, Springer-Verlag

24. Mehta S, Mirza AJ, Dunbar RP, Barei DP, Benirschke SK (2010) A staged treatment plan for the management of Type II and Type IIIA open calcaneus fractures. J Orthop Trauma 24(3):142-147

25. Mulcahy DM, McCormack DM, Stephens MM (1998) Intra-articular calcaneal fractures: effect of open reduction and internal fixation on the contact characteristics of the subtalar joint. Foot Ankle Int 19(12):842-848

26. Nehme A, Chaminade B, Chiron P, Fabie F, Tricoire $\mathrm{JL}$, Puget J (2004) Reduction et viaage percutanes des fractures thalamiques du calcaneus sous controle arthroscopique et fluoroscopique. Rev Chir Orthop Reparatrice Appar Mot 90:256-264

27. Nosewicz T, Knupp M, Barg A et al (2012) Mini-open sinus tarsi approach with percutaneous screw fixation of displaced calcaneal fractures: a prospective computed tomography-based study. Foot Ankle Int 33(11):925-933

28. Parisien JS, Vangsness T (1985) Arthroscopy oft he subtalar joint: an experimental approach. Arthroscopy 1:53-57

29. Peterson L, Romanus B, Dahlberg E (1976) Fracture oft he collum tali - an experimental study. J Biomechanics 9:227-279

30. Poeze M, Verbruggen JP, Brink PR (2008) The relationship between the outcome of operatively treated calcaneal fractures and institutional fracture load. A systematic review of the literature. J Bone Joint Surg Am 90(5):1013-1021

31. Radnay CS, Clare MP, Sanders RW (2009) Subtalar fusion after displaced intra-articular calcaneal frac tures: does initial operative treatment matter? J Bone Joint Surg Am 91:541-546

32. Rammelt S (2014) An update on the treatment of calcaneal fractures. J Orthop Trauma 28:549-550

33. Rammelt S, Goronzy J (2015) Subtalar dislocations. Foot Ankle Clin 20(2):253-264. doi:10.1016/j. fcl.2015.02.008

34. Rammelt S, Zwipp H (2004) Calcaneus fractures: facts, controversies and recent developments. Injury 35(5):443-461. (Review)

35. Rammelt S, Zwipp H (2009) Talar neck and body fractures. Injury 40:120-135

36. Rammelt S, Zwipp H (2014) Fractures of the calcaneus: current treatment strategies. Acta Chir Orthop Traumal Czech 81:177-196

37. Rammelt S, Gavlik JM, Barthel S, Zwipp H (2002) The value of subtalar arthroscopy in the management of intra-articular calcaneus fractures. Foot Ankle Int 23:906-916

38. Rammelt S, Amlang M, Barthel S, Zwipp H (2004) Minimally-invasive treatment of calcaneal fractures. Injury 35(Suppl 2):SB55-SB63. (Review)

39. Rammelt S, Amlang M, Barthel S, Gavlik JM, Zwipp $H$ (2010) Percutaneous treatment of less severe intrarticular calcaneal fractures. Clin Orthop Relat Res 468:983-990

40. Rammelt S, Dürr C, Schneiders W, Zwipp H (2012) Minimal-invasive Osteosynthese von Kalkaneusfrakturen. Oper Orthop Traumatol 24:383-395

41. Rammelt S, Winkler J, Zwipp H (2013) Osteosynthese zentraler Talusfrakturen. Oper Orthop Traumatol 25:525-541
42. Rammelt S, Zwipp H, Schneiders W, Dürr C (2013) Severity of injury predicts subsequent function in surgically treated displaced intraarticular calcaneal fractures. Clin Orthop Relat Res 471(9):2885-2898

43. Rübberdt A, Feil R, Stengel D et al (2006) Die klinische wertigkeit des ISO-C(3D) bei der Osteosynthese des Fersenbeins. Unfallchirurg 109(2):112118

44. Sanders R (1992) Intra-articular fractures of the calcaneus: present state of art. J Orthop Trauma 6:252-265

45. Sanders R (2000) Displaced intra-articular fractures of the calcaneus. J Bone Joint Surg Am 82(2):225250

46. Sanders R (2012) Turning tongues into joint depressions: a new calcaneal osteotomy. J Orthop Trauma 26(3):193-196

47. Sanders R, Rammelt $S$ (2013) Fractures of the calcaneus. In: Coughlin MJ, Saltzman CR, Anderson JB (Hrsg) Mann's surgery of the foot \& ankle, 9. Aufl. Elsevier, St. Louis, S 2041-2100

48. Sangeorzan BJ, Ananthakrishnan D, Tencer AF (1995) Contact characteristics of the subtalar joint after a simulated calcaneus fracture. J Orthop Trauma 9(3):251-258

49. Schepers $\mathrm{T}$ (2011) The sinus tarsi approach in displaced intra-articular calcaneal fractures: a systematic review. Int Orthop 35(5):697-703

50. Schuberth JM, Cobb MD, Talarico RH (2009) Minimally invasive arthroscopic-assisted reduction with percutaneous fixation in the management of intra-articular calcaneal fractures: a review of 24 cases. J Foot Ankle Surg 48:315-322

51. Schulze W, Richter J, Klapprich TH, Muhr G (1998) Funktionsergebnisse nach operativer Therapie von Talusfrakturen. Chirurg 69:1207-1213

52. Tezval M, Dumont C, Sturmer KM (2007) Prognostic reliability of the Hawkins sign in fractures of the talus. J Orthop Trauma 21:538-543

53. Vallier HA, Nork SE, Benirschke SK, Sangeorzan BJ (2003) Surgical treatment of talar body fractures. J Bone Joint Surg Am 85-A:1716-1724

54. Vallier HA, Reichard SG, Boyd AJ, Moore TA (2004) A new look at the Hawkins classicifcaiton for talar neck fractures: which features of injury and treatment are predictive of osteonecrosis? J Bone Joint Surg Am 86-A:1616-1624

55. Woon CY, Chong KW, Yeo W, Eng-Meng Yeo N, Wong MK (2011) Subtalar arthroscopy and flurosocopy in percutaneous fixation of intra-articular calcaneal fractures: the best of both worlds. J Trauma 71:917-925

56. Younger A (2013) A calcaneal fracture study illustrates a need for better statistical methods for orthopaedic outcomes: commentary on an article by Per-Henrik Ågren, MD, et al.:."Operative versus nonoperative treatment of displaced intra-articular calcaneal fractures. a prospective, randomized, controlled multicenter trial".J J Bone Joint Surg Am 95(15):e111

57. Zwipp H (1994) Chirurgie des Fußes. Springer, Wien

58. Zwipp H, Baumgart F, Cronier P et al (2004) integral classification of injuries (ICI) tot he bone, joints, and ligaments - application to injuries oft he foot. Injury 35(Suppl 2):SB3-SB9

59. Zwipp H, Rammelt S, Gavlik JM (2009) Anatomische Rekonstruktion fehlverheilter Talusfrakturen. Fuß Sprunggelenk 7:88-96

60. Zwipp H, Rammelt S, Amlang M, Pompach M, Dürr C (2013) Osteosynthese dislozierter intraartikulärer Calcaneusfrakturen. Oper Orthop Traumatol 25:554-568

61. Zwipp H, Rammelt S (2014) Tscherne Unfallchirurgie: Fuß. Springer-Verlag, Berlin 